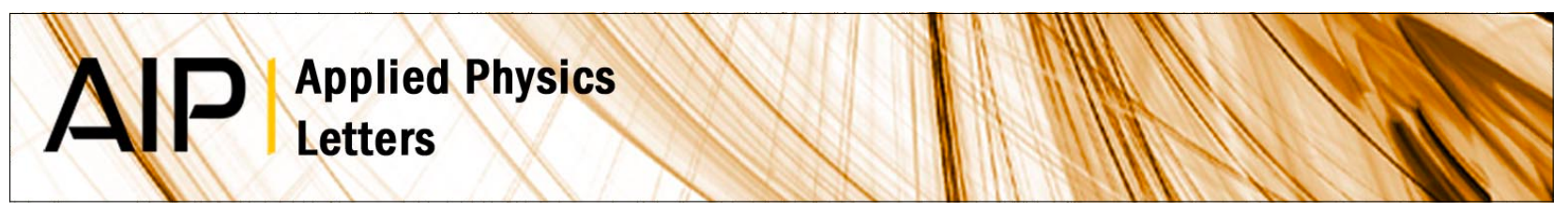

\title{
Non-volatile ferroelectric gating of magnetotransport anisotropy in $(\mathrm{Ga}, \mathrm{Mn})(\mathrm{As}, \mathrm{P})$
}

E. Mikheev, I. Stolichnov, Z. Huang, A. W. Rushforth, J. A. Haigh et al.

Citation: Appl. Phys. Lett. 100, 262906 (2012); doi: 10.1063/1.4731245

View online: http://dx.doi.org/10.1063/1.4731245

View Table of Contents: http://apl.aip.org/resource/1/APPLAB/v100/i26

Published by the American Institute of Physics.

\section{Related Articles}

Spin filtering in molecular junction: Magnetoresistance evaluation from wave-function calculations J. Chem. Phys. 138, 094105 (2013)

A graphene solution to conductivity mismatch: Spin injection from ferromagnetic metal/graphene tunnel contacts into silicon

J. Appl. Phys. 113, 17C502 (2013)

Magnetoresistance in inhomogeneous graphene/metal hybrids

J. Appl. Phys. 113, 083907 (2013)

Anomalous electronic and magnetic properties of the Eu2Ru2O7 pyrochlore

J. Appl. Phys. 113, 17E102 (2013)

Negative magnetoresistance and anomalous Hall effect in GeMnTe-SnMnTe spin-glass-like system J. Appl. Phys. 113, 063702 (2013)

\section{Additional information on Appl. Phys. Lett.}

Journal Homepage: http://apl.aip.org/

Journal Information: http://apl.aip.org/about/about_the_journal

Top downloads: http://apl.aip.org/features/most_downloaded

Information for Authors: http://apl.aip.org/authors

\section{ADVERTISEMENT}

\section{AIP Applied Physics Letters}

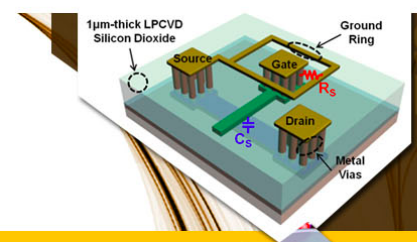

SUAFACES AND INTERFACES

Focusing on physical, chemical, biological, structural, optical, magnetic and electrical
properties of surfaces and interfaces, and more

\section{EXPLORE WHAT'S} NEW IN APL
ENERCY CONVERSION AND STORACE 


\title{
Non-volatile ferroelectric gating of magnetotransport anisotropy in $(\mathrm{Ga}, \mathrm{Mn})(\mathrm{As}, \mathrm{P})$
}

\author{
E. Mikheev, ${ }^{1}$ I. Stolichnov, ${ }^{1}$ Z. Huang, ${ }^{1}$ A. W. Rushforth, ${ }^{2}$ J. A. Haigh, ${ }^{2}$ R. P. Campion, ${ }^{2}$ \\ K. W. Edmonds, ${ }^{2}$ B. L. Gallagher, ${ }^{2}$ and N. Setter ${ }^{1}$ \\ ${ }^{1}$ Ceramics Laboratory, EPFL-Swiss Federal Institute of Technology, Lausanne 1015, Switzerland \\ ${ }^{2}$ School of Physics and Astronomy, University of Nottingham, Nottingham NG7 2RD, United Kingdom
}

(Received 14 May 2012; accepted 9 June 2012; published online 27 June 2012)

\begin{abstract}
We demonstrate charge-mediated and non-volatile control of anisotropic magnetoresistance $(\mathrm{AMR})$ in a dilute magnetic semiconductor $(\mathrm{Ga}, \mathrm{Mn})(\mathrm{As}, \mathrm{P})$ with an integrated polymer ferroelectric gate. The persistent electric field associated with switchable polarization in the ferroelectric layer is shown to be capable of strongly modulating the AMR magnitude. Furthermore, ferroelectric gate switching has a profound impact on the nature of AMR, changing the symmetry of the effect and enhancing/suppressing the crystalline component of AMR. Thus, in addition to a rather weak modulation of the ferromagnetic Curie temperature (4-5 K) reported previously, the ferroelectric gate can induce a strong deterministic switching of the magnetotransport anisotropy. (C) 2012 American Institute of Physics. [http://dx.doi.org/10.1063/1.4731245]
\end{abstract}

Anisotropic magnetoresistance (AMR), referring to the symmetrical dependence of electrical resistivity on magnetization vector orientation, draws a great deal of attention in view of applications in magnetic data storage. The dilute magnetic semiconductor (DMS) (Ga,Mn)As has recently emerged as a model system for AMR. Owing to a strong spin-orbit coupling and a relatively simple band diagram, it quickly became a preferred testing ground for first principle and phenomenological models. ${ }^{1-3}$

DMS are of particular interest for exploring AMRrelated phenomena because of the possibilities of external control of ferromagnetism, given the carrier-coupled nature of their ferromagnetism and the resulting susceptibility of the magnetic properties to electric field. A variety of manifestations of magneto-electric coupling in $(\mathrm{Ga}, \mathrm{Mn}) \mathrm{As}$ and related materials includes dielectric and ferroelectric gatebased control of ferromagnetic transition, ${ }^{4,5}$ coercive field ${ }^{5,6}$ and magnetization vector orientation. ${ }^{7}$ The magnitude of AMR in $(\mathrm{Ga}, \mathrm{Mn})$ As was also reported to be susceptible to modulation by electric field applied to a gate. ${ }^{8-10}$

In this work, we report on AMR in phosphorus codoped $(\mathrm{Ga}, \mathrm{Mn}) \mathrm{As}$, controlled via a polymer ferroelectric gate. We demonstrate that large changes in AMR magnitude can be accompanied by profound changes in its shape. In particular, ferroelectric polarization-induced suppression of the AMR and switching of the symmetry between dominant uniaxial and biaxial forms are demonstrated.

We use ferroelectric field effect transistor (FeFET) devices based on a 7-nm $\left(\mathrm{Ga}_{0.94} \mathrm{Mn}_{0.06}\right)\left(\mathrm{As}_{0.9} \mathrm{P}_{0.1}\right)$ magnetic active channel layer grown by molecular beam epitaxy (MBE) on a (001) GaAs substrate with a $25-\mathrm{nm} \mathrm{Ga}\left(\mathrm{As}_{0.9} \mathrm{P}_{0.1}\right)$ buffer layer. Further details of MBE growth can be found in Ref. 11 . 25/100-nm thick Ti/Au contacts were deposited by electron beam evaporation. A $180 \times 2000 \mu \mathrm{m}$ Hall bar channel oriented along the [1-10] orientation of $(\mathrm{Ga}, \mathrm{Mn})(\mathrm{As}, \mathrm{P})$ was defined by standard photolithography. The distance of $650 \mu \mathrm{m}$ separated two pairs of Hall voltage probe leads connected to the channel. Ferroelectric co-polymer P(VDF-TrFE) (77/23\%) film with thickness of $300 \pm 10 \mathrm{~nm}$ was spin coated from a $2 \%$ solution in methyl ethyl ketone, followed by a short 7 -min. long crystallization anneal at $136^{\circ} \mathrm{C}$. The top gate $\mathrm{Au}$ electrode was deposited by thermal evaporation. An additional $400 \times 400 \mu \mathrm{m}^{2}$ electrode has been patterned on each Hall bar mesa for independent control of the ferroelectric layer performance. The tests systematically deliver high quality sharp polarization hysteresis loops with remnant polarization of $8-9 \mu \mathrm{C} / \mathrm{cm}^{2}$ and coercive field of $550-650 \mathrm{kV} / \mathrm{cm}$, for the loop frequency of $10 \mathrm{kHz}$. The detailed analysis of the ferroelectric gate operation including polarization hysteresis data has been presented in the previous publication. ${ }^{12}$

The usage of phosphorus co-doped DMS and a minimized annealing schedule to avoid the removal of compensating defects ${ }^{13}$ results in devices which combine high resistive modulation with robust ferromagnetism. Reversal of the ferroelectric polarization by applying $\pm 14 \mathrm{~V}$ to the gate electrode results in a low temperature on/off ratio above 10 between the accumulation and depletion states (Fig. 1(a)). In the accumulation state, ferromagnetism below $31 \mathrm{~K}$ is revealed using the well-established technique ${ }^{14}$ based on the cusp of sheet resistance derivative $d R_{\text {sheet }} / d T$ (Fig. 1(b)). This cusp is suppressed in depletion due to low conductivity, but a 6-7 K downward shift of the ferromagnetic transition temperature $\mathrm{T}_{\mathrm{C}}$ can be confirmed via ferromagnetic switching signatures in magnetotransport as described elsewhere. ${ }^{15,16}$

The temperature dependence of resistance in ferroelectrically gated $(\mathrm{Ga}, \mathrm{Mn})(\mathrm{As}, \mathrm{P})$ and $(\mathrm{Ga}, \mathrm{Mn})$ As follows a thermal activation-type law at low temperatures (Fig. 1(c)):

$$
\left(R_{\text {sheet }} / R_{0}\right)^{-1}=\exp \left(\Delta E / k_{B} T\right)
$$

where $R_{\text {sheet }} / R_{0}$ is the sheet resistance normalized by the exponential pre-factor, $\Delta E$ is the activation energy, and $k_{B}$ is the Boltzmann constant. $(\mathrm{Ga}, \mathrm{Mn})(\mathrm{As}, \mathrm{P})$ annealing studies ${ }^{13}$ and comparison with an analogue reference device based on a 7-nm $\left(\mathrm{Ga}_{0.94} \mathrm{Mn}_{0.06}\right)$ As DMS (Fig. 1(b)) show that P doping induces a progressive metal-to-insulator transition (MIT), 
(a)
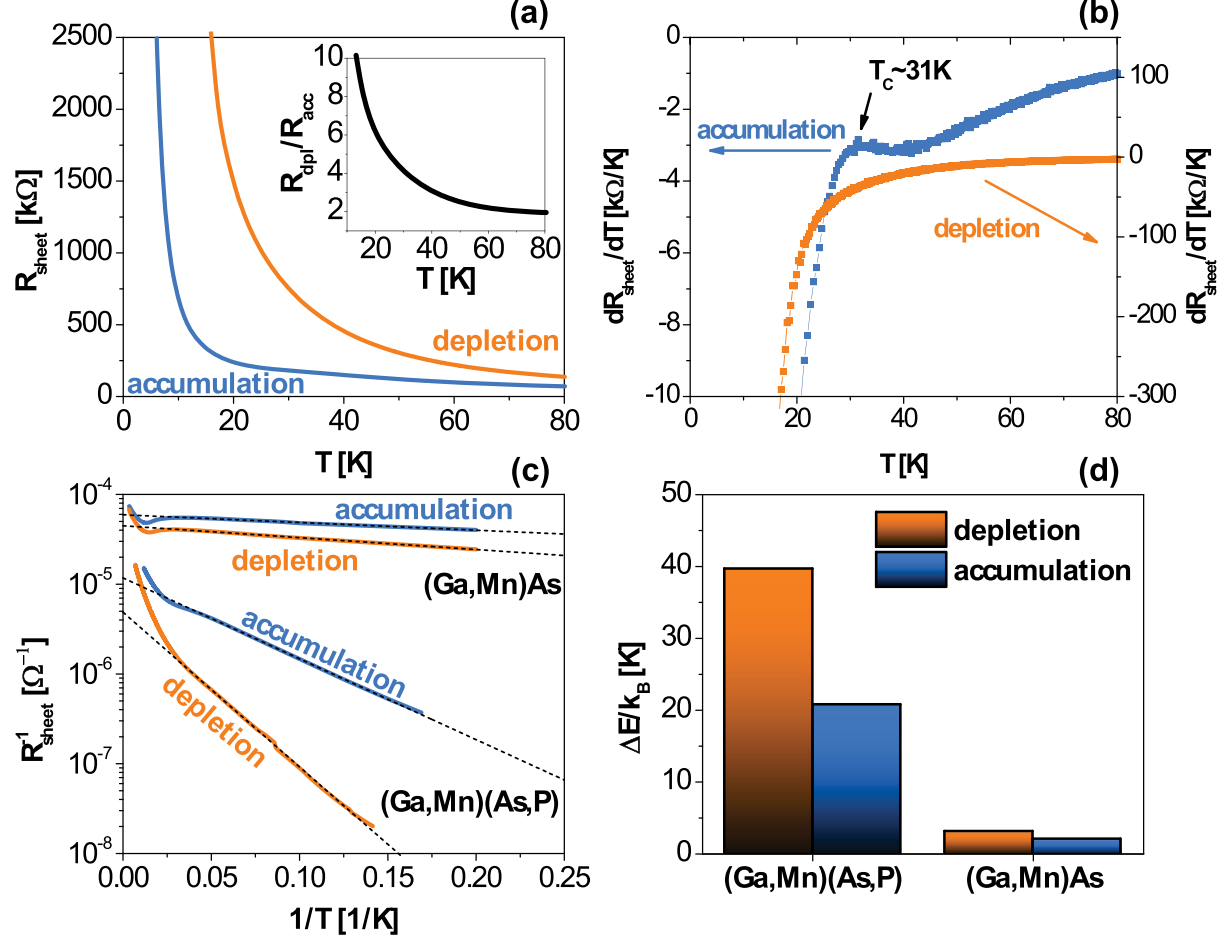

FIG. 1. Electrical transport in the (Ga,Mn) $(\mathrm{As}, \mathrm{P})$ layer, in accumulation and depletion states of the ferroelectric gate. (a) Temperature dependence of $R_{\text {sheet }}$ and the on/off ratio (inset). (b) Temperature derivative of $R_{\text {sheet }}$. (c) Illustration of thermal activation behavior and comparison to a reference device without phosphorus co-doping. (d) Extracted activation energies for the two ferroelectric gate states in both devices. linked to the Mn impurity moving deeper into the bandgap. ${ }^{17}$ The action of the ferroelectric gate allows one to move towards or away from the MIT, as evidenced by the increase of $\Delta E$ upon poling the gate in depletion (Fig. 1(d)).

Large $R_{\text {sheet }}$ and its modulation are interesting in view of obtaining large and gate controllable AMR. ${ }^{18}$ Fig. 2(a) shows measurements with a constant saturating magnetic field of $0.5 \mathrm{~T}$ rotated between the [001] out-of-plane (OOP) and [1-10] in-plane (IP) orientations. Here, the angular dependence of AMR is of second order in $\theta$, the field angle with respect to [1-10], as defined in Fig. 2(b), and can be described by:

$$
\frac{\Delta R_{X X}(\theta)}{\min \left(R_{X X}\right)}=\frac{A M R_{O O P}}{2} \cdot \cos (2 \theta),
$$

where $\Delta R_{X X} / \min \left(R_{X X}\right)$ is the change of longitudinal electrical resistance normalized by its minimum. $A M R_{O O P}$ is the conventional measure of AMR defined as the normalized difference of $R_{X X}$ with magnetization $M$ oriented along the current and out of plane vectors $\mathbf{I}$ and $\mathbf{n}$ :

$$
A M R_{O O P}=\frac{R_{X X}(\vec{M} \| \vec{I})-R_{X X}(\vec{M} \| \vec{n})}{R_{X X}(\vec{M} \| \vec{n})} .
$$

Fig. 2(c) shows a large enhancement of $A M R_{O O P}$ in depletion mode at temperatures below the ferromagnetic transition (near $30 \mathrm{~K}$ ). It reaches a factor of 3 at $15 \mathrm{~K}$. It was achieved owing to the large resistivity modulation in Fig. 1(a), as illustrated by the scaling of $A M R_{O O P}$ with the zerofield electrical resistance (Fig. 2(d)).

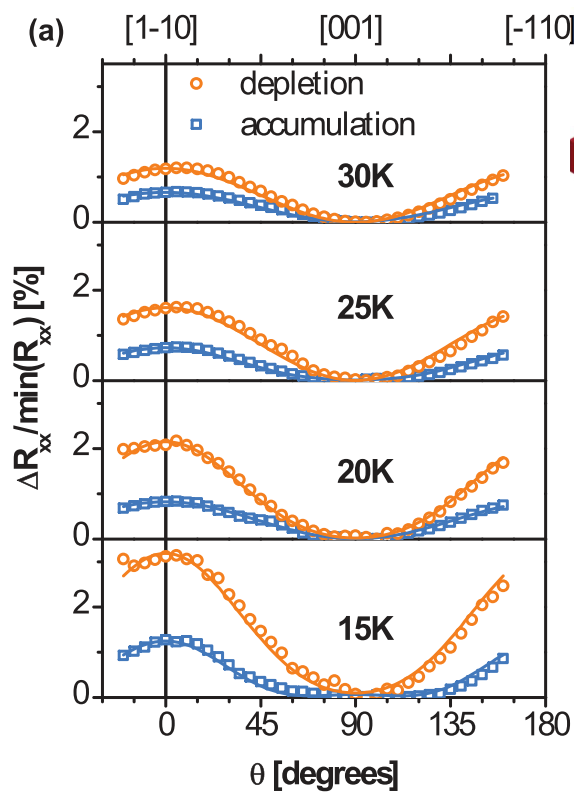

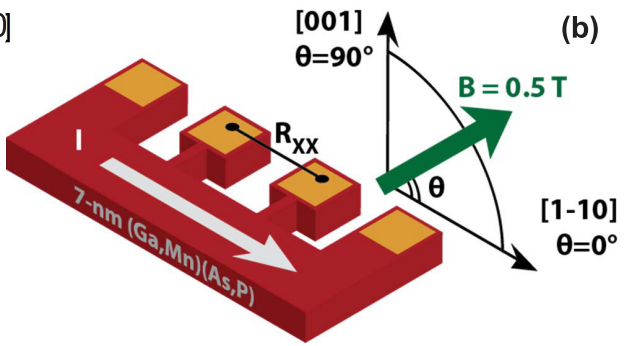

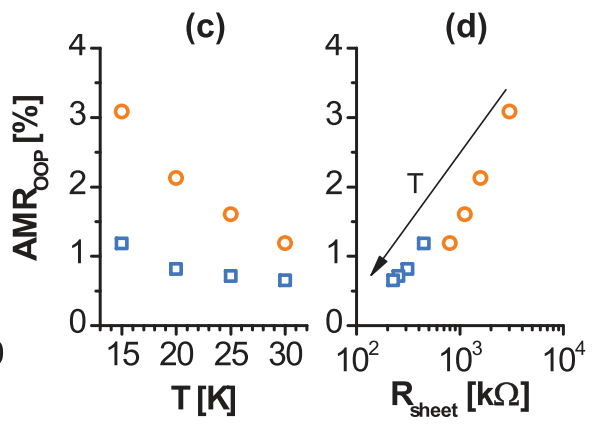

FIG. 2. (a) Ferroelectric gate modulation of AMR in a saturating field of $0.5 \mathrm{~T}$, rotated in the out-of-plane geometry, illustrated in (b). (c) Temperature dependence of the AMR magnitude and (d) its scaling with the zerofield sheet resistance of the $(\mathrm{Ga}, \mathrm{Mn})(\mathrm{As}, \mathrm{P})$ channel. 

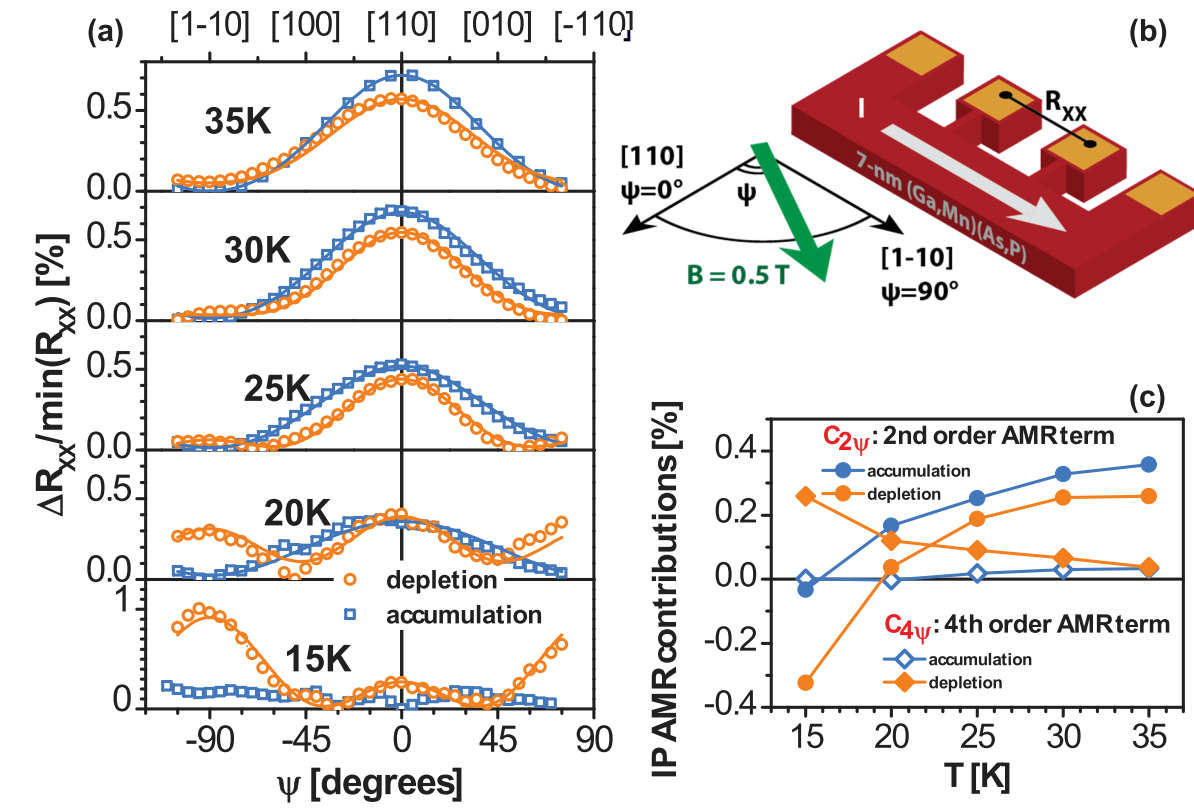

(b)

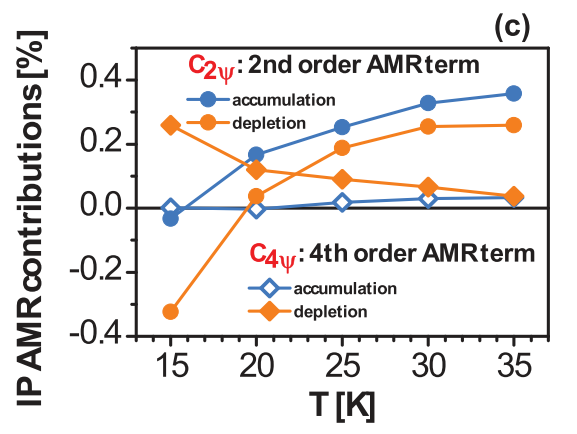

FIG. 3. (a) Ferroelectric switching of the AMR symmetry in a saturating field of $0.5 \mathrm{~T}$, rotated in the sample plane, as illustrated in (b). (c) Temperature dependence of the 2 nd and 4 th order contributions $\left(C_{2 \psi}\right.$ and $C_{4 \psi}$ ) to the in-plane AMR.

A noteworthy aspect of the data in Fig. 2(a) is the positive sign of $A M R_{O O P}$ across the entire studied temperature range, i.e., $R_{X X}$ is higher for $\vec{M} \| \vec{I}$ than for $\vec{M} \| \vec{n}$. This is in contrast to the negative AMR commonly observed in compressively strained (Ga,Mn)As films. ${ }^{19-21}$ The negative AMR is consistent with the strain inversion to tensile in $(\mathrm{Ga}, \mathrm{Mn})(\mathrm{As}, \mathrm{P})$ grown on $\mathrm{Ga}(\mathrm{As}, \mathrm{P})$, as predicted by microscopic modeling based on the k.p theory combined with mean-field kinetic-exchange splitting. ${ }^{22,23}$

The most striking manifestations of gate effect on AMR occur in a different measurement geometry, with a constant $0.5 \mathrm{~T}$ field rotated in the sample plane. The field angle $\psi$ is defined with respect to the [110] orientation, as shown in Fig. 3(a). This in-plane AMR is a superposition of 2nd and 4th order contributions $C_{2 \psi}$ and $C_{4 \psi}::^{1}$

$$
\frac{\Delta R_{X X}(\psi)}{\min \left(R_{X X}\right)}=C_{2 \psi} \cdot \cos (2 \psi)+C_{4 \psi} \cdot \cos (4 \psi) .
$$

This provides an accurate description for the observed intricate angular dependence of the in-plane AMR, shown for the 15-35 K range in Fig. 3(b). Here, the gate effect induces very pronounced changes in AMR shape. It is further illustrated in Fig. 3(c), where the values of $C_{2 \psi}$ and $C_{4 \psi}$ extracted by fitting Eq. (4) to the experimental data are shown. Poling the ferroelectric gate modulates the 2nd order component and deterministically switches the 4 th order one on and off.

This leads to a particularly remarkable situation at $\mathrm{T}=20 \mathrm{~K}$, where the near perfect 2-fold AMR symmetry in accumulation is broken in depletion, where the $C_{4 \psi}$ component dominates. Equally noteworthy is the gated suppression of AMR at T $=15 \mathrm{~K}$, where in accumulation AMR is not distinguishable above thermal noise, while in depletion it reaches a large $1 \%$ amplitude.

The origin of the heavily modulated 4-th order component lies in the crystalline AMR, to be distinguished from the non-crystalline AMR, which dominates in bulk ( $\mathrm{Ga}, \mathrm{Mn}) \mathrm{As}$. The crystalline components of the AMR arise from modulations of the shape of the Fermi surface and the interplay with $\mathrm{k}$-dependent scattering times as the magnetization is rotated in strongly spin-orbit coupled systems. The effect on the Fermi surface of modulating carrier density by electrical gating will be larger when the Fermi energy is closer to the band edge, as is the case when $\mathrm{P}$ is co-doped with (Ga,Mn)As and carriers are compensated in the as-grown state by interstitial Mn defects. ${ }^{13}$ Also, localized states which can be strongly anisotropic may play a significant role in the transport properties of these highly resistive samples. Previously, large crystalline components have been observed to emerge in ultrathin DMS layers below 10-nm thickness. ${ }^{1,18}$ Control of crystalline components in ultrathin films has previously been demonstrated via strain using piezoelectric transducers ${ }^{24,25}$ and photolithographic constrictions. ${ }^{25,26}$ Here, we show a high degree of electrostatic control over the crystalline contribution to AMR induced by switchable polarization of the ferroelectric gate.

In conclusion, electric-field-controlled persistent switching of the AMR behavior has been observed in the ferroelectric FET structure with ultra-thin $(\mathrm{Ga}, \mathrm{Mn})(\mathrm{As}, \mathrm{P})$ channel. The essential features include the possibility of non-volatile, ferroelectric-gate-driven modulation/suppression of the AMR signal, and switching of the AMR symmetry with turning on/off the crystalline AMR component. From the application prospective, these results show a different mode of multiferroic device operation compared to the earlier reported ferroelectric control of ferromagnetic $\mathrm{T}_{\mathrm{C}}{ }^{5}$ In contrast to a relatively weak ferromagnetic $T_{C}$ modulation, the AMR switching presented in this letter has a deterministic character, implying potential applications in memories and logic elements. These results reveal great opportunities offered by ferroelectric gating that brings to DMS-based spintronic devices additional functionalities of non-volatile switching and reversible, non-destructive nano-patterning of ferroelectric domains.

The research at EPFL has received funding from the Swiss National Science Foundation Grant No. 132724 and the European Research Council under the European Union's 
Seventh Framework Programme (FP7/2007-2013)/ERC Grant Agreement No. [268058]. A.W.R. acknowledges support from a UK EPSRC Career Acceleration Fellowship (EP/H003487/1). The authors thank Dr. Karel Vyborny and Professor J. Trodahl for helpful discussions.

${ }^{1}$ A. W. Rushforth, K. Výborný, C. S. King, K. W. Edmonds, R. P. Campion, C. T. Foxon, J. Wunderlich, A. C. Irvine, P. Vašek, V. Novák, K. Olejník, J. Sinova, T. Jungwirth, and B. L. Gallagher, Phys. Rev. Lett. 99, 147207 (2007).

${ }^{2}$ K. Výborný, J. Kučera, J. Sinova, A. Rushforth, B. Gallagher, and T. Jungwirth, Phys. Rev. B 80, 165204 (2009).

${ }^{3}$ M. Trushin, K. Výborný, P. Moraczewski, A. Kovalev, J. Schliemann, and T. Jungwirth, Phys. Rev. B 80, 134405 (2009).

${ }^{4}$ H. Ohno, D. Chiba, F. Matsukura, T. Omiya, E. Abe, T. Dietl, Y. Ohno, and K. Ohtani, Nature (London) 408, 944-946 (2000).

${ }^{5}$ I. Stolichnov, S. W. E. Riester, H. J. Trodahl, N. Setter, A. W. Rushforth, K. W. Edmonds, R. P. Campion, C. T. Foxon, B. L. Gallagher, and T. Jungwirth, Nature Mater. 7, 464-467 (2008).

${ }^{6}$ D. Chiba, M. Yamanouchi, F. Matsukura, and H. Ohno, Science (N.Y.) 301, 943-5 (2003).

${ }^{7}$ D. Chiba, M. Sawicki, Y. Nishitani, Y. Nakatani, F. Matsukura, and H. Ohno, Nature (London) 455, 515-518 (2008).

${ }^{8}$ K. Olejník, M. Owen, V. Novák, J. Mašek, A. Irvine, J. Wunderlich, and T. Jungwirth, Phys. Rev. B 78, 054403 (2008)

${ }^{9}$ M. H. S. Owen, J. Wunderlich, V. Novák, K. Olejník, J. Zemen, K. Výborný, S. Ogawa, a C. Irvine, a J. Ferguson, H. Sirringhaus, and T. Jungwirth, New J. Phys. 11, 023008 (2009).

${ }^{10} \mathrm{P}$. Balestrière, T. Devolder, J.-V. Kim, P. Lecoeur, J. Wunderlich, V. Novák, T. Jungwirth, and C. Chappert, Appl. Phys. Lett. 99, 242505 (2011).

${ }^{11}$ A. W. Rushforth, M. Wang, N. R. S. Farley, R. P. Campion, K. W. Edmonds, C. R. Staddon, C. T. Foxon, and B. L. Gallagher, J. Appl. Phys. 104, 073908 (2008).
Appl. Phys. Lett. 100, 262906 (2012)

${ }^{12}$ I. Stolichnov, S. W. E. Riester, E. Mikheev, N. Setter, a W. Rushforth, K. W. Edmonds, R. P. Campion, C. T. Foxon, B. L. Gallagher, T. Jungwirth, and H. J. Trodahl, Nanotechnology 22, 254004 (2011).

${ }^{13}$ A. Casiraghi, A. W. Rushforth, M. Wang, N. R. S. Farley, P. Wadley, J. L. Hall, C. R. Staddon, K. W. Edmonds, R. P. Campion, C. T. Foxon, and B. L. Gallagher, Appl. Phys. Lett. 97, 122504 (2010).

${ }^{14}$ V. Novák, K. Olejník, J. Wunderlich, M. Cukr, K. Výborný, a. W. Rushforth, K. W. Edmonds, R. P. Campion, B. L. Gallagher, J. Sinova, and T. Jungwirth, Phys. Rev. Lett. 101, 077201 (2008).

${ }^{15}$ G. Xiang, A. Holleitner, B. Sheu, F. Mendoza, O. Maksimov, M. Stone, P. Schiffer, D. Awschalom, and N. Samarth, Phys. Rev. B 71, 241307 (2005).

${ }^{16}$ G. Xiang and N. Samarth, Phys. Rev. B 76, 054440 (2007).

${ }^{17}$ J. Mašek, J. Kudrnovský, F. Máca, J. Sinova, A. MacDonald, R. Campion, B. Gallagher, and T. Jungwirth, Phys. Rev. B 75, 045202 (2007).

${ }^{18}$ R. R. Gareev, a. Petukhov, M. Schlapps, J. Sadowski, and W. Wegscheider, Appl. Phys. Lett. 96, 052114 (2010).

${ }^{19}$ D. Baxter, D. Ruzmetov, J. Scherschligt, Y. Sasaki, X. Liu, J. Furdyna, and C. Mielke, Phys. Rev. B 65, 212407 (2002).

${ }^{20} \mathrm{~K}$. Wang, K. Edmonds, R. Campion, L. Zhao, C. Foxon, and B. Gallagher, Phys. Rev. B 72, 085201 (2005).

${ }^{21}$ W. Limmer, M. Glunk, J. Daeubler, T. Hummel, W. Schoch, R. Sauer, and S. T. B. Goennenwein, Phys. Rev. B 74, 205205 (2006).

${ }^{22}$ T. Jungwirth, M. Abolfath, J. Sinova, J. Kučera, and A. H. MacDonald, Appl. Phys. Lett. 81, 4029 (2002).

${ }^{23}$ T. Jungwirth, Curr. Appl. Phys. 3, 461-464 (2003).

${ }^{24}$ A. W. Rushforth, E. De Ranieri, J. Zemen, J. Wunderlich, K. W. Edmonds, C. S. King, E. Ahmad, R. P. Campion, C. T. Foxon, B. L. Gallagher, K. Výborný, J. Kučera, and T. Jungwirth, Phys. Rev. B 78, 085314 (2008).

${ }^{25}$ E. De Ranieri, A. W. Rushforth, K. Výborný, U. Rana, E. Ahmad, R. P. Campion, C. T. Foxon, B. L. Gallagher, a C. Irvine, J. Wunderlich, and T. Jungwirth, New J. Physics 10, 065003 (2008).

${ }^{26}$ J. Shiogai, D. Schuh, W. Wegscheider, M. Kohda, J. Nitta, and D. Weiss, Appl. Phys. Lett. 98, 083101 (2011). 